\title{
A twistor space action for Yang-Mills theory
}

\author{
Alexander D. Popov $\odot^{*}$ \\ Institut für Theoretische Physik, Leibniz Universität Hannover, \\ Appelstraße 2, 30167 Hannover, Germany
}

(Received 29 March 2021; accepted 28 June 2021; published 26 July 2021)

\begin{abstract}
We consider the twistor space $\mathcal{P}^{6} \cong \mathbb{R}^{4} \times \mathbb{C} P^{1}$ of $\mathbb{R}^{4}$ with a nonintegrable almost complex structure $\mathcal{J}$ such that the canonical bundle of the almost complex manifold $\left(\mathcal{P}^{6}, \mathcal{J}\right)$ is trivial. It is shown that $\mathcal{J}$ holomorphic Chern-Simons theory on a real (6|2)-dimensional graded extension $\mathcal{P}^{6 / 2}$ of the twistor space $\mathcal{P}^{6}$ is equivalent to self-dual Yang-Mills theory on Euclidean space $\mathbb{R}^{4}$ with Lorentz invariant action. It is also shown that adding a local term to a Chern-Simons-type action on $\mathcal{P}^{6 / 2}$, one can extend it to a twistor action describing full Yang-Mills theory.
\end{abstract}

DOI: 10.1103/PhysRevD.104.026015

\section{INTRODUCTION}

Let $M^{4}$ be an oriented real four-manifold with a Riemannian metric and $P\left(M^{4}, \mathrm{SO}(4)\right)$ the principal bundle of orthonormal frames over $M^{4}$. The twistor space $\operatorname{Tw}\left(M^{4}\right)$ of $M^{4}$ can be defined as an associated bundle [1]

$$
\operatorname{Tw}\left(M^{4}\right)=P \times_{\mathrm{SO}(4)} \mathrm{SO}(4) / \mathrm{U}(2)
$$

with the canonical projection

$$
\pi: \operatorname{Tw}\left(M^{4}\right) \rightarrow M^{4} .
$$

Fibers of this bundle are two-spheres $S_{x}^{2} \cong \mathrm{SO}(4) / \mathrm{U}(2)$ which parametrize complex structures $J_{x}$ on the tangent space $T_{x} M^{4}$ at $x \in M^{4}$ compatible with a Euclidean metric and orientation of $M^{4}$. It means that $J_{x} \in \operatorname{End}\left(T_{x} M^{4}\right)$ with $J_{x}^{2}=-\mathrm{Id}$ and $J_{x}$ is an isometry of $T_{x} M^{4}$ preserving orientation.

An almost complex structure $J$ on $M^{4}$ is a global section of the bundle (1.2). Note that while a manifold $M^{4}$ admits in general no almost complex structure (e.g., four-sphere $S^{4}$ ), its twistor space $\operatorname{Tw}\left(M^{4}\right)$ can always be equipped with two natural almost complex structures. The first, $\mathcal{J}=\mathcal{J}_{+}$, introduced in [1], is integrable if and only if the Weyl tensor of Riemannian metric on $M^{4}$ is self-dual, while the second, $\mathcal{J}=\mathcal{J}_{-}$, introduced in [2], is nonintegrable (and never integrable), i.e., the Nijenhuis tensor of $\mathcal{J}$ does not vanish.

\footnotetext{
*alexander.popov@itp.uni-hannover.de
}

Published by the American Physical Society under the terms of the Creative Commons Attribution 4.0 International license. Further distribution of this work must maintain attribution to the author(s) and the published article's title, journal citation, and DOI. Funded by SCOAP .
Twistor space $\mathcal{P}^{6}=\operatorname{Tw}\left(\mathbb{R}^{4}\right) \cong \mathbb{R}^{4} \times S^{2}$ of $\mathbb{R}^{4}$ with an almost complex structure $\mathcal{J}$ is a particular case of almost complex six-manifolds to be discussed in this paper. Twistor space $\left(\mathcal{P}^{6}, \mathcal{J}\right)$ is a complex manifold $\mathcal{P}_{\mathbb{C}}^{3}$ for integrable $\mathcal{J}$ and it is an almost complex manifold with an SU(3)-structure and nonvanishing torsion for nonintegrable $\mathcal{J}$. Twistor literature focuses on complex twistor space $\mathcal{P}_{\mathbb{C}}^{3}$ (see, e.g., [3-5]) and very rarely on the nonintegrable case (see, e.g., $[2,6,7])$.

The goal of twistor theory is to take some unconstraint analytic object on $\operatorname{Tw}\left(M^{4}\right)$ (e.g., Dolbeault cohomology classes) and transform them to objects on $M^{4}$ which will be constrained by some differential equations $[3,4]$. In particular, the self-dual Yang-Mills (SDYM) equations on Euclidean space $\mathbb{R}^{4}$ can be described as field equations of holomorphic Chern-Simons theory defining holomorphic bundles on the complex twistor space $\mathcal{P}_{\mathbb{C}}^{3}$ via the PenroseWard correspondence [3-5]. This correspondence can be extended to the nonintegrable case (see, e.g., $[6,7]$ ).

The field equations of $\mathcal{J}$-holomorphic Chern-Simons $(\mathcal{J}$-hCS $)$ theory on $\left(\mathcal{P}^{6}, \mathcal{J}\right)$ read

$$
\mathcal{F}^{0,2}=P^{0,1} P^{0,1} \mathcal{F}=(\mathrm{d} \mathcal{A}+\mathcal{A} \wedge \mathcal{A})^{0,2}=0,
$$

where $P^{0,1}=\frac{1}{2}(\mathrm{Id}+\mathrm{i} \mathcal{J})$ is the projector onto $(0,1)$-part of one-forms, $\mathcal{A}$ is a connection one-form on a complex vector bundle $\mathcal{E}$ over $\left(\mathcal{P}^{6}, \mathcal{J}\right)$ and $\mathcal{F}=\mathrm{d} \mathcal{A}+\mathcal{A} \wedge \mathcal{A}$ is the curvature of $\mathcal{A}$. One can expect that Eqs. (1.3) are obtained by variation of the action functional

$$
\begin{aligned}
S= & \frac{\mathrm{i}}{8} \int_{\mathcal{P}^{6}} \Omega \wedge \mathrm{CS}(\mathcal{A})^{0,3}=\frac{\mathrm{i}}{8} \int_{\mathcal{P}^{6}} \\
& \Omega \wedge \operatorname{tr}\left(\mathcal{A} \wedge \mathrm{d} \mathcal{A}+\frac{2}{3} \mathcal{A} \wedge \mathcal{A} \wedge \mathcal{A}\right)^{0,3},
\end{aligned}
$$


where $\Omega$ is a $(3,0)$-form with respect to $\mathcal{J}$ on $\left(\mathcal{P}^{6}, \mathcal{J}\right)$, i.e., $\Omega$ is a global section of the canonical bundle of $\left(\mathcal{P}^{6}, \mathcal{J}\right)$. However, the canonical bundle of $\mathcal{P}_{\mathbb{C}}^{3} \cong \mathbb{C} P^{3} \backslash \mathbb{C} P^{1}$ is the nontrivial holomorphic line bundle $\mathcal{O}(-4)$ with the first Chern class -4 . Hence, there is no nonsingular holomorphic volume form $\Omega$ on $\mathcal{P}_{\mathbb{C}}^{3}$. Thus, the functional (1.4) is not defined on $\mathcal{P}_{\mathbb{C}}^{3}$.

The triviality of the canonical bundle can be restored if instead of $\mathcal{P}_{\mathbb{C}}^{3}$ one considers the supertwistor space $\mathcal{P}_{\mathbb{C}}^{3 / 4} \cong$ $\mathbb{C} P^{3 / 4} \backslash \mathbb{C} P^{1 / 4}$ with four holomorphic fermionic dimensions, each of type $\Pi \mathcal{O}(1)$ bundle, where the operator $\Pi$ inverts the Grassmann parity of fibre coordinates. The canonical bundle of $\mathcal{P}_{\mathbb{C}}^{3 / 4}$ is trivial and hence there is a holomorphic volume form $\tilde{\Omega}$ on $\mathcal{P}_{\mathbb{C}}^{3 / 4}$. This fact was used by Witten for introducing twistor string theory and holomorphic ChernSimons theory (hCS) on $\mathcal{P}_{\mathbb{C}}^{3 / 4}[8]$. The action of hCS theory on $\mathcal{P}_{\mathbb{C}}^{3 / 4}$ can be written in the form (1.4) after substituting $\widetilde{\Omega}$ instead of $\Omega$ and integrating over $\mathcal{P}_{\mathbb{C}}^{3 \mid 4}$. The field equations will be (1.3) with $\mathcal{A}^{0,1}=P^{0,1} \mathcal{A}$ depending on four Grassmann variables taking values in the bundle $\Pi \mathcal{O}(1) \otimes$ $\mathbb{C}^{4}$ over $\mathcal{P}_{\mathbb{C}}^{3}$. This hCS theory on $\mathcal{P}_{\mathbb{C}}^{3 / 4}$ in turn is equivalent [8] to self-dual subsector of $\mathcal{N}=4$ supersymmetric YangMills theory on $\mathbb{R}^{4}$ (see, e.g., [9-11] for reviews and references) in the form of Chalmers and Siegel [12]. The $\mathcal{N}=4$ SDYM equations can be truncated to the bosonic SDYM equations [12] and on the twistor level this was discussed, e.g., in [13-15].

Despite the success of the supertwistor description of supersymmetric Yang-Mills theories, there was a desire to get a twistor description of pure bosonic SDYM theory. Recently, it was proposed by Costello to work with hCS theory on the bosonic twistor space $\mathcal{P}_{\mathbb{C}}^{3}$ by allowing $\Omega$ in (1.4) to be meromorphic instead of holomorphic [16]. After choosing a meromorphic form $\Omega$ on $\mathcal{P}_{\mathbb{C}}^{3}$ and imposing some boundary conditions on fields at poles of $\Omega$, one can reduce the action (1.4) to the $4 d$ action for SDYM theory as it was demonstrated in $[16,17]$. Depending on the gauge choice, the twistor action is reduced to the action for group-valued fields $[18,19]$ or to the action for Lie-algebra valued fields $[20,21]$, both of which are well known in the literature. However, the choice of (3,0)-form $\Omega$ and of its singularities is not unique and different choices lead to a range of actions on $\mathbb{R}^{4}$, not all of which have equations of motion equivalent to the SDYM equations [17].

All the above-mentioned actions break Lorentz invariance. The actions [18-21] for the SDYM equations were discussed long time ago by Chalmers and Siegel in [12], where it was shown that these $4 d$ actions at more than one loop generate diagrams that do not relate to quantum YangMills theory. These flaws are absent for the ChalmersSiegel $4 d$ action which is a truncation (a limit of small coupling constant) of the standard Yang-Mills action. We want to obtain this $4 d$ action in the framework of twistor approach. We show that this is possible by using a nonintegrable almost complex structure $\mathcal{J}$ on the twistor space
$\mathcal{P}^{6}$ such that the canonical bundle becomes trivial and hence there exists a globally defined $(3,0)$-form $\Omega$ on $\left(\mathcal{P}^{6}, \mathcal{J}\right)$ which can be used in (1.4).

The action [12] contains gauge field coupled with a propagating anti-self-dual auxiliary field $G_{\dot{\alpha} \dot{\beta}}=\varepsilon^{\alpha \beta} G_{\alpha \dot{\alpha}, \beta \dot{\beta}}$ with $\dot{\alpha}, \dot{\beta}=1,2$. The field $G_{\dot{\alpha} \dot{\beta}}$ corresponds to additional degrees of freedom parametrized by some cohomology groups on the complex twistor space $\mathcal{P}_{\mathbb{C}}^{3}[11,22]$ and can be obtained from the component $\mathcal{A}^{0,1}$ along $\mathbb{C} P^{1} \hookrightarrow \mathcal{P}_{\mathbb{C}}^{3 \mid 4}$ in hCS theory on the supertwistor space (see, e.g., [11] and references therein). This $G_{\dot{\alpha} \dot{\beta}}$ enters into the $\mathcal{N}=4$ SDYM supermultiplet $\left(f_{\alpha \beta}, \chi^{\alpha i}, \phi^{i j}, \tilde{\chi}_{\dot{\alpha} i}, G_{\dot{\alpha} \dot{\beta}}\right)$, where the fields have helicities $\left(+1,+\frac{1}{2}, 0,-\frac{1}{2},-1\right), i=1, \ldots, 4$. Truncations of the self-dual $\mathcal{N}=4$ super-Yang-Mills to the case $\mathcal{N}<4$, including the bosonic case $\mathcal{N}=0$, can be obtained by considering weighted projective supertwistor space $[10,14]$ or exotic supertwistor space $[9,15]$. The approach similar to that in $[14,15]$ can be used in the case of twistor space $\left(\mathcal{P}^{6}, \mathcal{J}\right)$ with nonintegrable almost complex structure $\mathcal{J}$ on $\mathcal{P}^{6}$. We will show that the $4 d$ ChalmersSiegel action [12] can be obtained from an action functional for $\mathcal{J}$-hCS theory on a graded twistor space $\mathcal{P}^{6 / 2}$ with two real fermionic directions, each parametrizing trivial real line bundle over $\left(\mathcal{P}^{6}, \mathcal{J}\right)$. The Chern-Simons type action on $\mathcal{P}^{6 \mid 2}$ is introduced by using globally defined form $\tilde{\Omega}=$ $\Omega \wedge \mathrm{d} \eta_{1} \wedge \mathrm{d} \eta_{2}$ on $\mathcal{P}^{6 \mid 2}$, where $\Omega$ is a global section of the trivial canonical bundle of $\mathcal{P}^{6}$. Components of gauge potential $\mathcal{A}$ in this theory take values in the Grassmann algebra $\Lambda\left(\mathbb{R}^{2}\right)$ generated by two real scalars $\eta_{1}, \eta_{2}$. We also show that this action can be extended to a twistor action describing full Yang-Mills theory on $\mathbb{R}^{4}$ after adding some local terms to $\mathcal{J}$-hCS Lagrangian on the twistor space $\mathcal{P}^{6 \mid 2}$.

\section{SELF-DUAL YANG-MILLS AND TWISTORS}

\section{A. Almost complex structures on $\operatorname{Tw}\left(M^{4}\right)$}

We defined the twistor space $\operatorname{Tw}\left(M^{4}\right)$ of a Riemannian manifold $M^{4}$ as the associated bundle (1.1) of complex structures $J_{x}$ on tangent spaces $T_{x} M^{4}$. Global sections of the projection (1.2) are identified, if such sections exist, with almost complex structures $J$ on $M^{4}$, i.e., with tensors $J=$ $\left(J_{\mu}^{\nu}\right) \in \operatorname{End}\left(T M^{4}\right)$ such that $J_{\mu}^{\sigma} J_{\sigma}^{\nu}=-\delta_{\mu}^{\nu}, \mu, \nu=1, \ldots, 4$.

While a manifold $M^{4}$ has in general no almost complex structures, its twistor space $Q^{6}:=\operatorname{Tw}\left(M^{4}\right)$ can be always provided in a natural way with an almost complex structure $\mathcal{J}$, a tensor on $Q^{6}$ with $\mathcal{J}^{2}=-$ Id. In fact, the Levi-Civita connection on $M^{4}$ generates the splitting of the tangent bundle $T Q^{6}$ into the direct sum

$$
T Q^{6}=V \oplus H
$$

of vertical and horizontal subbundles of $T Q^{6}$. The space $V_{q}$ in $q \in Q^{6}$ is tangent to the fibre $\pi^{-1}(\pi(q))$ over $x=\pi(q) \in$ $M^{4}$ of the projection $\pi: Q^{6} \rightarrow M^{4}$. Recall that the fiber over $x=\pi(q)$ is identified with $S_{x}^{2} \cong \mathrm{SO}(4) / \mathrm{U}(2)$ and so it has 
a natural complex structure $J^{v}$. Hence, we can define an almost complex structure $\mathcal{J}$ on $Q^{6}$ using the decomposition (2.1) by setting

$$
\mathcal{J}=\mathcal{J}^{\text {int }}=\mathcal{J}^{v} \oplus \mathcal{J}^{h},
$$

where $\mathcal{J}^{h}$ is an almost complex structure equal in the point $q \in Q^{6}$ to the complex structure $\mathcal{J}_{q}^{h}$ on $H_{q} \cong T_{\pi(q)} M^{4}=T_{x} M^{4}$. Thus, the twistor space $Q^{6}$ has a natural almost complex structure $\mathcal{J}$.

It was shown in [1] that if the Weyl tensor of $M^{4}$ is selfdual then the almost complex structure (2.2) on $Q^{6}$ is integrable and $\left(Q^{6}, \mathcal{J}^{\text {int }}\right)$ inherits the structure of a complex analytic 3-manifold $Q_{\mathbb{C}}^{3}$. It was also shown in [2] that

$$
\mathcal{J}=\mathcal{J}^{\text {non }}=\mathcal{J}^{v} \oplus\left(-\mathcal{J}^{h}\right)
$$

is an almost complex structure on $Q^{6}$ which is never integrable. These structures differ in sign along $M^{4}$.

\section{B. Twistor correspondence}

Let $E$ be a rank $k$ complex vector bundle over $M^{4}$ and $A$ a connection one-form (gauge potential) on $E$ with the curvature $F=\mathrm{d} A+A \wedge A$ (gauge field). The gauge field $F$ is called self-dual if it satisfies the equations

$$
* F=F \Leftrightarrow \frac{1}{2} \varepsilon_{\mu \nu \lambda \sigma} F^{\lambda \sigma}=F_{\mu \nu},
$$

where $*$ denotes the Hodge star operator, $\varepsilon_{\mu \nu \lambda \sigma}$ is the completely antisymmetric tensor on $M^{4}$ with $\varepsilon_{1234}=1$ in the Riemannian metric $\mathrm{d} s^{2}=\delta_{\mu \nu} e^{\mu} e^{\nu}$ for an orthonormal basis $\left\{e^{\mu}\right\}$ on $T^{*} M^{4}$.

Bundles $E$ with self-dual connections $A$ are called selfdual. It was proven in [1] that the self-dual bundle $E$ over self-dual manifold $M^{4}$ lifts to a holomorphic bundle $\mathcal{E}$ over the complex twistor space $Q_{\mathbb{C}}^{3}=\left(\operatorname{Tw}\left(M^{4}\right), \mathcal{J}^{\text {int }}\right)$ and $\mathcal{E}$ is holomorphically trivial on fibers $\mathbb{C} P_{x}^{1}$ of projections $\pi: Q^{6} \rightarrow M^{4}$ for each $x \in M^{4}$. The bundle $\mathcal{E}=\pi^{*} E$ is defined by the connection $\mathcal{A}=\pi^{*} A$ such that its curvature $\mathcal{F}=\mathrm{d} \mathcal{A}+\mathcal{A} \wedge \mathcal{A}$ satisfies the Eqs. (1.3) and $\mathcal{F}=\pi^{*} F$ is the pull-back to $\mathcal{E}$ of self-dual gauge field $F$ on $E \rightarrow M^{4}$. Vice versa, solutions to the holomorphic Chern-Simons field equations (1.3) on the twistor space $Q_{\mathbb{C}}^{3}$, with $\mathcal{F}_{\mid \mathbb{C} P_{x}^{1}}=$ 0 for any $x \in M^{4}$, give solutions to the SDYM equations (2.4) on $M^{4}$. The map between solutions to the SDYM equations on $M^{4}$ and solutions to the hCS field equations on $Q_{\mathbb{C}}^{3}=\left(\operatorname{Tw}\left(M^{4}\right), \mathcal{J}^{\text {int }}\right)$ is called the Penrose-Ward transform.

For nonintegrable almost complex structure (2.3) on $Q^{6}$ the manifold $\left(Q^{6}, \mathcal{J}^{\text {non }}\right)$ is not complex. However, on $\left(Q^{6}, \mathcal{J}^{\text {non }}\right)$ one can introduce bundles with $\mathcal{J}$-holomorphic structure (pseudo-holomorphic bundles) [23]. Let $\mathcal{E}$ be a complex rank $k$ vector bundle over $Q^{6}$ endowed with a connection $\mathcal{A}$. According to Bryant [23], a connection $\mathcal{A}$ on
$\mathcal{E}$ is said to define a $\mathcal{J}$-holomorphic structure if it has curvature $\mathcal{F}$ of type $(1,1)$ with respect to $\mathcal{J}$, i.e.,

$$
\mathcal{F}^{0,2}=0 .
$$

It is not difficult to show that twistor correspondence between solutions of SDYM equations (2.4) on $M^{4}$ and solutions of $\mathcal{J}$-hCS equations (2.5) on the almost complex twistor space $\left(Q^{6}, \mathcal{J}\right)$ still persists (see, e.g., [7]). This will be discussed in more details later for the case of flat Euclidean space $M^{4}=\mathbb{R}^{4}$.

\section{TWISTOR SPACE OF $\mathbb{R}^{4}$}

According to the definition (1.1), twistor space of $\mathbb{R}^{4}$ is $\mathcal{P}^{6}:=\operatorname{Tw}\left(\mathbb{R}^{4}\right) \cong \mathbb{R}^{4} \times S^{2}$. Due to diffeomorphism with $\mathbb{R}^{4} \times S^{2}$, the manifold $\mathcal{P}^{6}$ is fibered not only over $\mathbb{R}^{4}$,

$$
\pi: \mathcal{P}^{6} \stackrel{S^{2}}{\longrightarrow} \mathbb{R}^{4},
$$

but also over $S^{2}$,

$$
\mathcal{P}^{6} \stackrel{\mathbb{R}^{4}}{\longrightarrow} S^{2},
$$

with spaces $\mathbb{R}^{4}$ as fibres.

\section{A. Almost complex structures $\mathcal{J}$}

In Sec. II we described generic construction of an almost complex structure $\mathcal{J}$ on a twistor space $\operatorname{Tw}\left(M^{4}\right)$. Here, we give explicit form of $\mathcal{J}$ for the case $M^{4}=\mathbb{R}^{4}$.

Recall that a complex structure $J$ on $\mathbb{R}^{4}$ is a tensor $J=$ $\left(J_{\mu}^{\nu}\right)$ such that $J_{\mu}^{\sigma} J_{\sigma}^{\nu}=-\delta_{\mu}^{\nu}$. All constant complex structures on $\mathbb{R}^{4}$ are parametrized by the two-sphere $S^{2} \cong$ $\mathrm{SO}(4) / \mathrm{U}(2) \cong \mathrm{SU}(2) / \mathrm{U}(1)$ defined by the equation

$$
\delta_{a b} s^{a} s^{b}=1
$$

for $s^{a} \in \mathbb{R}^{3}, a, b=1,2,3$. One can choose generic $J$ in the form

$$
J_{\mu}^{\nu}=s_{a} \bar{\eta}_{\mu \sigma}^{a} \delta^{\sigma \nu}
$$

where

$$
\bar{\eta}_{\mu \nu}^{a}=\left\{\varepsilon_{b c}^{a}, \mu=b, \nu=c ;-\delta_{\mu}^{a}, \nu=4 ; \delta_{\nu}^{a}, \mu=4\right\}
$$

are antisymmetric 't Hooft tensors, $\mu, \nu=1, \ldots, 4$. Using the identities

$$
\bar{\eta}_{\mu \sigma}^{a} \bar{\eta}_{\sigma \nu}^{b}=-\delta^{a b} \delta_{\mu \nu}-\varepsilon^{a b c} \bar{\eta}_{\mu \nu}^{c},
$$

one can show that $J^{2}=-$ Id. Here, we consider $\mathbb{R}^{4}$ as a space with the metric $\mathrm{d} s_{\mathbb{R}^{4}}^{2}=\delta_{\mu \nu} \mathrm{d} x^{\mu} \mathrm{d} x^{\nu}$, where $x^{\mu}$ are coordinates on $\mathbb{R}^{4}$. 
Let $\left\{e^{\alpha}\right\}$ represents an orthonormal coframe on $S^{2}$, i.e.,

$$
\mathrm{d} s_{S^{2}}^{2}=\delta_{\alpha \beta} e^{\alpha} e^{\beta}
$$

for $\alpha, \beta=1,2$. The canonical form of complex structure $\dot{j}$ on $S^{2}$ is

$$
\dot{\mathfrak{i}}=\left(\mathfrak{j}_{\alpha}^{\beta}\right) \quad \text { with } \quad \dot{\mathfrak{j}}_{1}^{2}=-\dot{\mathfrak{j}}_{2}^{1}=1 \Rightarrow \dot{\mathfrak{l}}_{\alpha}^{\sigma} \mathfrak{j}_{\sigma}^{\beta}=-\delta_{\alpha}^{\beta} .
$$

It is obvious that both

$$
\mathcal{J}=\mathcal{J}^{\text {int }}=(J, \mathfrak{j})
$$

and

$$
\mathcal{J}=\mathcal{J}^{\text {non }}=(-J, \dot{\mathfrak{i}})
$$

are almost complex structures on the twistor space $\mathcal{P}^{6}$ of $\mathbb{R}^{4}$. Complex twistor space $\mathcal{P}_{\mathbb{C}}^{3}=\left(\mathcal{P}^{6}, \mathcal{J}\right)$ with integrable almost complex structure $\mathcal{J}=\mathcal{J}^{\text {int }}$ has been studied a lot in the literature and in the following we will focus on nonintegrable almost complex structure $\mathcal{J}=\mathcal{J}^{\text {non }}$.

\section{B. Complex coordinates for $\mathcal{J}=\mathcal{J}^{\text {int }}$}

The two-sphere $S^{2}$, global coordinates $s^{a}$ on which are used in (3.4), is conformally equivalent to $\mathbb{R}^{2}$. One can cover $S^{2}$ by two patches $U_{ \pm} \cong \mathbb{R}^{2}$ with local coordinates

$v_{+}^{\alpha}=\frac{s^{\alpha}}{1+s^{3}}$ on $U_{+} \quad$ and $\quad v_{-}^{\alpha}=\frac{s^{\alpha}}{1-s^{3}}$ on $U_{-}$,

in which the metric on $S^{2}$ is conformally flat,

$$
\begin{aligned}
\mathrm{d} s_{S^{2} \mid U_{ \pm}}^{2} & =\delta_{\alpha \beta} e_{ \pm}^{\alpha} e_{ \pm}^{\beta}=\frac{4 \delta_{\alpha \beta} \mathrm{d} v_{ \pm}^{\alpha} \mathrm{d} v_{ \pm}^{\beta}}{\left(1+\rho_{ \pm}^{2}\right)^{2}} \quad \text { with } \\
\rho_{ \pm}^{2} & =\delta_{\alpha \beta} v_{ \pm}^{\alpha} v_{ \pm}^{\beta} .
\end{aligned}
$$

On the intersection of two patches we have

$$
v_{+}^{\alpha}=\rho_{+}^{2} v_{-}^{\alpha},
$$

where $\alpha, \beta=1,2$.

On $S^{2}$ one can introduce vector fields of type $(1,0)$ and $(0,1)$ with respect to $\dot{i}$ from $(3.8)$,

$$
\begin{aligned}
& \frac{\partial}{\partial \lambda_{ \pm}} \text {and } \frac{\partial}{\partial \bar{\lambda}_{ \pm}}, \\
& \dot{\mathfrak{i}}\left(\partial_{\lambda_{ \pm}}\right)=\mathrm{i} \partial_{\lambda_{ \pm}} \quad \text { and } \quad \dot{j}\left(\partial_{\bar{\lambda}_{ \pm}}\right)=-\mathrm{i} \partial_{\bar{\lambda}_{ \pm}},
\end{aligned}
$$

where

$$
\lambda_{ \pm}=v_{ \pm}^{1}+\mathrm{i} v_{ \pm}^{2} \text { and } \lambda_{+}=\lambda_{-}^{-1} \text { on } U_{+} \cap U_{-}
$$

are complex coordinates on $U_{ \pm} \subset S^{2}$. One-forms, dual to the vector fields (3.14), are $\mathrm{d} \lambda_{ \pm}$and $\mathrm{d} \bar{\lambda}_{ \pm}$. Sphere $\left(S^{2}, \dot{\mathrm{j}}\right)$ with the coordinates (3.15) can be identified with the Riemann sphere $\mathbb{C} P^{1}$.

By using the complex structure (3.4) on $\mathbb{R}^{4}$, one can introduce a $\mathbb{C} P^{1}$-family of complex coordinates on $\mathbb{R}^{4}$ given by formulas

$$
w_{+}^{1}=y^{1}+\lambda_{+} \bar{y}^{2} \quad \text { and } \quad w_{+}^{2}=y^{2}-\lambda_{+} \bar{y}^{1},
$$

where

$$
\begin{aligned}
& y^{1}=x^{1}+\mathrm{i} x^{2}, \quad y^{2}=x^{3}-\mathrm{i} x^{4}, \\
& \bar{y}^{1}=x^{1}-\mathrm{i} x^{2}, \quad \text { and } \quad \bar{y}^{2}=x^{3}+\mathrm{i} x^{4} .
\end{aligned}
$$

The coordinates (3.15) together with (3.16) provide complex coordinates on $\mathcal{P}^{6}$ given by

$$
w_{+}^{1}, \quad w_{+}^{2} \quad \text { and } \quad w_{+}^{3}=\lambda_{+} \quad \text { on } \quad \mathcal{U}_{+}=U_{+} \times \mathbb{R}^{4} \subset \mathcal{P}^{6}
$$

and

$$
\begin{aligned}
& w_{-}^{1}=\lambda_{-} y^{1}+\bar{y}^{2}, \\
& w_{-}^{2}=\lambda_{-} y^{2}-\bar{y}^{1} \text { and } \\
& w_{-}^{3}=\lambda_{-} \text {on } \mathcal{U}_{-}=U_{-} \times \mathbb{R}^{4} \subset \mathcal{P}^{6} .
\end{aligned}
$$

On the intersection $\mathcal{U}_{+} \cap \mathcal{U}_{-}$of patches $\mathcal{U}_{ \pm} \subset \mathcal{P}^{6}$ these coordinates are related by formulas

$$
w_{+}^{\alpha}=w_{+}^{3} w_{-}^{\alpha} \quad \text { and } \quad w_{+}^{3}=\frac{1}{w_{-}^{3}} \text { on } \mathcal{U}_{+} \cap \mathcal{U}_{-} .
$$

Hence, the transition functions relating $w_{+}^{a}$ and $w_{-}^{a}$ are holomorphic functions on $\mathcal{U}_{+} \cap \mathcal{U}_{-}, a=1,2$, 3. This means that $\mathcal{J}^{\text {int }}$ is an integrable almost complex structure and $\mathcal{P}_{\mathbb{C}}^{3}=\left(\mathcal{P}^{6}, \mathcal{J}^{\text {int }}\right)$ is a complex 3-manifold. From (3.16)-(3.18) it follows that the manifold $\mathcal{P}_{\mathbb{C}}^{3}$ can be identified with the total space of the holomorphic vector bundle over $\mathbb{C} P^{1}$,

$$
\mathcal{P}_{\mathbb{C}}^{3}=\mathcal{O}(1) \oplus \mathcal{O}(1) \rightarrow \mathbb{C} P^{1},
$$

with coordinates $w_{ \pm}^{\alpha}$ on fibers $\mathbb{C}_{J}^{2}$ over points $J \in \mathbb{C} P^{1}$ parametrized by $\lambda_{ \pm} \subset U_{ \pm} \subset \mathbb{C} P^{1}$.

\section{Complex coordinates for $\mathcal{J}=\mathcal{J}^{\text {non }}$}

By using the almost complex structure (3.10), we can introduce complex coordinates

$$
\begin{aligned}
& z_{+}^{1}=\bar{w}_{+}^{1}=\bar{y}^{1}+\bar{\lambda}_{+} y^{2}, \quad z_{+}^{2}=\bar{w}_{+}^{2}=\bar{y}^{2}-\bar{\lambda}_{+} y^{1}, \\
& z_{+}^{3}=w_{+}^{3}=\lambda_{+} \text {on } \mathcal{U}_{+} \subset \mathcal{P}^{6}
\end{aligned}
$$


and

$$
\begin{aligned}
& z_{-}^{1}=\bar{w}_{-}^{1}=\bar{\lambda}_{-} \bar{y}^{1}+y^{2}, \quad z_{-}^{2}=\bar{w}_{-}^{2}=\bar{\lambda}_{-} \bar{y}^{2}-y^{1}, \\
& z_{-}^{3}=w_{-}^{3}=\lambda_{-} \text {on } \mathcal{U}_{-} \subset \mathcal{P}^{6} .
\end{aligned}
$$

On the intersection $\mathcal{U}_{+} \cap \mathcal{U}_{-}$of two coordinate patches $\mathcal{U}_{ \pm} \subset \mathcal{P}^{6}=\mathcal{U}_{+} \cup \mathcal{U}_{-}$we have

$$
z_{+}^{\alpha}=\bar{z}_{+}^{3} z_{-}^{\alpha} \quad \text { and } \quad z_{+}^{3}=\frac{1}{z_{-}^{3}} .
$$

From (3.22) we see that the transition functions on $\mathcal{U}_{+} \cap$ $\mathcal{U}_{-}$are not holomorphic. This reflects nonintegrability of the almost complex structure (3.10). From (3.22) it follows that the manifold $\left(\mathcal{P}^{6}, \mathcal{J}\right)$ with $\mathcal{J}=\mathcal{J}^{\text {non }}$ can be identified with the total space of the antiholomorphic vector bundle

$$
\overline{\mathcal{O}}(1) \oplus \overline{\mathcal{O}}(1) \rightarrow \mathbb{C} P^{1}
$$

over $\mathbb{C} P^{1}$. Both base and fibers $\overline{\mathbb{C}}_{J}^{2}$ of this bundle are complex spaces but they do not glue into a complex manifold for $\mathcal{J}$ given by (3.10).

\section{Spinor notation}

The rotation group $\mathrm{SO}(4)$ of space $\mathbb{R}^{4}$ is locally isomorphic to the group $\mathrm{SU}(2) \times \mathrm{SU}(2)$, where both groups $\mathrm{SU}(2)$ have two-dimensional fundamental (spinor) representations

$$
\mu=\left(\mu_{\alpha}\right) \text { and } \lambda=\left(\lambda_{\dot{\alpha}}\right) .
$$

Commuting components $\lambda_{\dot{\alpha}}$ of the spinor $\lambda$ are homogeneous coordinates on the Riemannian sphere $\mathbb{C} P^{1}$ such that

$\frac{\lambda_{\dot{2}}}{\lambda_{\mathrm{i}}}=\lambda_{+}$on $U_{+} \subset \mathbb{C} P^{1}$ and $\frac{\lambda_{\mathrm{i}}}{\lambda_{\dot{2}}}=: \lambda_{-}$on $U_{-} \subset \mathbb{C} P^{1}$.

Obviously, $\lambda_{+}=\lambda_{-}^{-1}$ if $\lambda_{\mathrm{i}} \neq 0$ and $\lambda_{\dot{2}} \neq 0$.

Isomorphism $\mathrm{SO}(4) \cong \mathrm{SU}(2) \times \mathrm{SU}(2)$ allows also to introduce spinor notation for complex coordinates on $\mathbb{R}^{4}$ by formula

$$
\begin{aligned}
\left(x^{\alpha \dot{\alpha}}\right) & =\left(\begin{array}{cc}
x^{1 \mathrm{i}} & x^{12} \\
x^{21} & x^{2 \dot{2}}
\end{array}\right)=\left(\begin{array}{cc}
y^{1} & -\bar{y}^{2} \\
y^{2} & \bar{y}^{1}
\end{array}\right) \\
& =\left(\begin{array}{cc}
x^{1}+\mathrm{i} x^{2} & -\left(x^{3}+\mathrm{i} x^{4}\right) \\
x^{3}-\mathrm{i} x^{4} & x^{1}-\mathrm{i} x^{2}
\end{array}\right) .
\end{aligned}
$$

From (3.26) it follows that

$$
x^{1 \mathrm{i}}=\bar{x}^{2 \dot{2}} \quad \text { and } \quad x^{1 \dot{2}}=-\bar{x}^{2 \dot{1}},
$$

where the overbar denotes complex conjugation. By using (3.26), one can rewrite (3.16) and (3.20) as follows

$$
w_{+}^{\alpha}=x^{\alpha \dot{\alpha}} \lambda_{\dot{\alpha}}^{+} \quad \text { and } \quad z_{+}^{\alpha}=-\dot{\mathfrak{j}}_{\beta}^{\alpha} x^{\beta \dot{\beta}} \hat{\lambda}_{\dot{\beta}}^{+},
$$

where

$$
\begin{aligned}
& \left(\lambda_{\dot{\alpha}}^{+}\right)=\frac{1}{\lambda_{\dot{1}}}\left(\lambda_{\dot{\alpha}}\right)=\left(\begin{array}{c}
1 \\
\lambda_{+}
\end{array}\right) \text {and } \\
& \left(\hat{\lambda}_{\dot{\alpha}}^{+}\right)=\left(\begin{array}{cc}
0 & -1 \\
1 & 0
\end{array}\right)\left(\bar{\lambda}_{\dot{\alpha}}^{+}\right)=\left(\begin{array}{c}
-\bar{\lambda}_{+} \\
1
\end{array}\right) .
\end{aligned}
$$

By definition, we have $\lambda_{\dot{\alpha}}^{-}=\lambda_{+}^{-1} \lambda_{\dot{\alpha}}^{+}$. and $\hat{\lambda}_{\dot{\alpha}}^{-}=\bar{\lambda}_{+}^{-1} \hat{\lambda}_{\dot{\alpha}}^{+}$.

\section{E. Vector fields and one-forms}

On the twistor space $\left(\mathcal{P}^{6}, \mathcal{J}\right)$ with $\mathcal{J}$ from (3.10) we have the natural basis $\left\{\frac{\partial}{\partial z^{a}}\right\}$ for the space of $(1,0)$ vector fields. On the intersection we have

$\frac{\partial}{\partial z_{+}^{\alpha}}=\bar{z}_{-}^{3} \frac{\partial}{\partial z_{-}^{\alpha}} \quad$ and $\quad \frac{\partial}{\partial z_{+}^{3}}=-\left(z_{-}^{3}\right)^{2} \frac{\partial}{\partial z_{-}^{3}}-z_{-}^{3} \bar{z}_{-}^{\alpha} \frac{\partial}{\partial \bar{z}_{-}^{\alpha}}$.

Using formulas (3.28), we can express these vector fields in terms of coordinates $\left(x^{\alpha \mathrm{i}}, \lambda_{ \pm}\right)$and their complex conjugates according to

$$
\begin{aligned}
& \frac{\partial}{\partial z_{ \pm}^{\alpha}}=-\gamma_{ \pm} \dot{\mathfrak{l}}_{\alpha}^{\beta} \lambda_{ \pm}^{\dot{\beta}} \frac{\partial}{\partial x^{\beta \dot{\beta}}}=:-\mathfrak{j}_{\alpha}^{\beta} V_{\beta}^{ \pm}, \\
& \frac{\partial}{\partial z_{+}^{3}}=\frac{\partial}{\partial \lambda_{+}}+\gamma_{+} \dot{\mathfrak{l}}_{\alpha}^{\beta} x^{\alpha \dot{1}} V_{\beta}^{+}, \\
& \frac{\partial}{\partial z_{-}^{3}}=\frac{\partial}{\partial \lambda_{-}}-\gamma_{-} \dot{\mathfrak{l}}_{\alpha}^{\beta} x^{\alpha \dot{2}^{2}} V_{\dot{\beta}}^{-},
\end{aligned}
$$

where we have used

$\lambda_{ \pm}^{\dot{\alpha}}=\varepsilon^{\dot{\alpha} \dot{\beta}} \lambda_{\dot{\beta}}^{ \pm} \quad$ with $\quad \varepsilon^{i \dot{2}}=-\varepsilon^{\dot{2} i}=1 \quad$ and

$\gamma_{ \pm}=\frac{1}{1+\lambda_{ \pm} \bar{\lambda}_{ \pm}}=\frac{1}{\hat{\lambda}_{ \pm}^{\dot{\alpha}} \lambda_{\dot{\alpha}}^{ \pm}}$

together with the convention $\varepsilon_{1 \dot{2}}=-\varepsilon_{\dot{2} \mathrm{i}}=-1$, which implies $\varepsilon_{\dot{\alpha} \dot{\beta}} \varepsilon^{\dot{\beta} \dot{\gamma}}=\delta_{\dot{\alpha}}^{\dot{y}}$. Thus, the vector fields

$V_{\alpha}^{ \pm}=\gamma_{ \pm} \lambda_{ \pm}^{\dot{\alpha}} \partial_{\alpha \dot{\alpha}}, \quad V_{3}^{+}=\gamma_{+}^{-2} \partial_{\lambda_{+}} \quad$ and $\quad V_{3}^{-}=\bar{\lambda}_{+}^{-2} V_{3}^{+}$

can be chosen as a basis of vector fields of type $(1,0)$ on $\mathcal{U}_{ \pm} \subset \mathcal{P}^{6}$ in the coordinates $\left(x^{\alpha \dot{\alpha}}, \lambda_{ \pm}, \bar{\lambda}_{ \pm}\right)$. Complex conjugate of (3.33) provide us with the vector fields 
$\bar{V}_{\alpha}^{ \pm}=\gamma_{ \pm} \dot{i}_{\alpha}^{\beta} \hat{\lambda}_{ \pm}^{\dot{\beta}} \partial_{\beta \dot{\beta}}, \quad \bar{V}_{3}^{+}=\gamma_{+}^{-2} \partial_{\bar{\lambda}_{+}} \quad$ and $\quad \bar{V}_{3}^{-}=\lambda_{+}^{-2} \bar{V}_{3}^{+}$

which form a basis of vector fields of type $(0,1)$ on $\mathcal{U}_{ \pm} \subset \mathcal{P}^{6}$.

It is easy to check that the basis of $(1,0)$ - and $(0,1)$-forms on $\mathcal{U}_{ \pm}$, which are dual to the vector fields (3.33) and (3.34), is given by forms

$$
\begin{aligned}
& E_{ \pm}^{\alpha}=-\left(\mathrm{d} x^{\alpha \dot{\alpha}}\right) \hat{\lambda}_{\dot{\alpha}}^{+}, \\
& E_{+}^{3}=\gamma_{+}^{2} \mathrm{~d} \lambda_{+} \quad \text { and } \\
& E_{-}^{3}=\bar{\lambda}_{+}^{-2} E_{+}^{3}, \quad \bar{E}_{ \pm}^{\alpha}=-\dot{\mathrm{j}}_{\beta}^{\alpha}\left(\mathrm{d} x^{\beta \dot{\beta}}\right) \lambda_{\dot{\beta}}, \\
& \bar{E}_{+}^{3}=\gamma_{+}^{2} \mathrm{~d} \bar{\lambda}_{+} \quad \text { and } \quad \bar{E}_{-}^{3}=\lambda_{+}^{-2} \bar{E}_{+}^{3} .
\end{aligned}
$$

One can easily verify that

$$
\mathrm{d}_{\mathcal{U}_{ \pm}}=\mathrm{d} z_{ \pm}^{a} \frac{\partial}{\partial z_{ \pm}^{a}}+\mathrm{d} \bar{z}_{ \pm}^{a} \frac{\partial}{\partial \bar{z}_{ \pm}^{a}}=E_{ \pm}^{a} V_{a}^{ \pm}+\bar{E}_{ \pm}^{a} \bar{V}_{a}^{ \pm}
$$

\section{F. Geometry of $\left(\mathcal{P}^{6}, \mathcal{J}\right)$}

We consider the twistor space $\left(\mathcal{P}^{6}, \mathcal{J}\right)$ with $\mathcal{J}$ from (3.10) and coordinates $\left\{z_{ \pm}^{a}\right\}$ on $\mathcal{U}_{ \pm} \subset \mathcal{P}^{6}$ given by (3.20)(3.22). In the following we often omit the signs \pm in coordinates, vector fields, one-forms etc. by considering all formula on the patch $\mathcal{U}_{+} \subset \mathcal{P}^{6}$.

By direct calculations we obtain that nonzero commutators of vector fields (3.33) and (3.34) are

$$
\begin{aligned}
& {\left[V_{3}, V_{\alpha}\right]=-\gamma^{-1} \dot{\mathbf{i}}_{\alpha}^{\beta} \bar{V}_{\beta}, \quad\left[V_{3}, \bar{V}_{\alpha}\right]=-\bar{\lambda} \gamma^{-1} \bar{V}_{\alpha},} \\
& {\left[V_{3}, \bar{V}_{3}\right]=2 \gamma\left(\bar{\lambda} \bar{V}_{3}-\lambda V_{3}\right),}
\end{aligned}
$$

$\left[\bar{V}_{3}, \bar{V}_{\alpha}\right]=-\gamma^{-1} \mathfrak{j}_{\alpha}^{\beta} V_{\beta} \quad$ and $\quad\left[\bar{V}_{3}, V_{\alpha}\right]=-\lambda \gamma^{-1} V_{\alpha}$,

where we used the formulas

$$
\partial_{\lambda}\left(\gamma \lambda^{\dot{\alpha}}\right)=\gamma^{2} \hat{\lambda}^{\dot{\alpha}} \quad \text { and } \quad \partial_{\bar{\lambda}}\left(\gamma \hat{\lambda}^{\dot{\alpha}}\right)=-\gamma^{2} \lambda^{\dot{\alpha}} .
$$

To prove integrability of an almost complex structure $\mathcal{J}$ one has to show that commutators of vector fields of type $(0,1)$ with respect to $\mathcal{J}$ will again be vector fields of type $(0,1)$ [24]. From (3.37) we see that this is not the case and therefore $\mathcal{J}$ is not integrable. For one-forms (3.35) we have

$$
\begin{aligned}
& \mathrm{d} E^{1}=\lambda \gamma^{-1} \bar{E}^{3} \wedge E^{1}+\gamma^{-1} \bar{E}^{2} \wedge \bar{E}^{3}, \\
& \mathrm{~d} E^{2}=\lambda \gamma^{-1} \bar{E}^{3} \wedge E^{2}+\gamma^{-1} \bar{E}^{3} \wedge \bar{E}^{1}, \\
& \mathrm{~d} E^{3}=-2 \lambda \gamma^{-1} \bar{E}^{3} \wedge E^{3},
\end{aligned}
$$

and complex conjugate formulas. The first terms in (3.40) define a torsionful connection on $\mathcal{P}^{6}$ with values in $u(1) \subset$ $s u(3)$ and the last terms define the Nijenhuis tensor (torsion) with nonvanishing components

$$
N_{\overline{2} \overline{3}}^{1}=\gamma^{-1}, \quad N_{\overline{3} \overline{1}}^{2}=\gamma^{-1}
$$

plus their complex conjugate $N_{23}^{\overline{1}}=N_{31}^{\overline{2}}=\gamma^{-1}$. From (3.40) we again see that $\left(\mathcal{P}^{6}, \mathcal{J}\right)$ is not a complex manifold but the total space (3.23) of the anti-holomorphic bundle over $\mathbb{C} P^{1}$. Furthermore, from $(3.40)$ we see that $\left(\mathcal{P}^{6}, \mathcal{J}\right)$ has an SU(3)-structure and the globally defined $(3,0)$-form $\Omega$ with

$\Omega=E_{+}^{1} \wedge E_{+}^{2} \wedge E_{+}^{3}=E_{-}^{1} \wedge E_{-}^{2} \wedge E_{-}^{3}$ on $\mathcal{U}_{+} \cap \mathcal{U}_{-}$

since

$$
E_{+}^{\alpha}=\bar{\lambda}_{+} E_{-}^{\alpha} \quad \text { and } \quad E_{+}^{3}=\bar{\lambda}_{+}^{-2} E_{-}^{3} .
$$

Hence, the canonical bundle of $\left(\mathcal{P}^{6}, \mathcal{J}\right)$ is trivial. From (3.40) it follows that

$$
\begin{gathered}
\mathrm{d}(\operatorname{Im} \Omega)=0, \\
\mathrm{~d}(\operatorname{Re} \Omega)=-\gamma^{-1}\left(E^{1} \wedge \bar{E}^{1}+E^{2} \wedge \bar{E}^{2}\right) \wedge E^{3} \wedge \bar{E}^{3},
\end{gathered}
$$

i.e., the real part of $\Omega$ is not closed. For the volume form on $\mathcal{P}^{6}$ we have

$$
\operatorname{Vol}_{6}=\frac{\mathrm{i}}{8} \Omega \wedge \bar{\Omega}=-\frac{\mathrm{i}}{2} \mathrm{~d}^{4} x \wedge \frac{\mathrm{d} \lambda \wedge \mathrm{d} \bar{\lambda}}{(1+\lambda \bar{\lambda})^{2}}
$$

where $\mathrm{d}^{4} x=\mathrm{d} x^{1} \wedge \mathrm{d} x^{2} \wedge \mathrm{d} x^{3} \wedge \mathrm{d} x^{4}$ in the coordinates (3.26).

\section{G. Twistor correspondence}

To conclude this section we describe a twistor correspondence between the SDYM model on $\mathbb{R}^{4}$ and $\mathcal{J}$-hCS theory on $\left(\mathcal{P}^{6}, \mathcal{J}\right)$.

Consider a complex vector bundle $E$ over $\mathbb{R}^{4}$ with a connection $A=A_{\alpha \dot{\alpha}} \mathrm{d} x^{\alpha \dot{\alpha}}$ and the covariant derivative $\nabla=\mathrm{d} x^{\alpha \dot{\alpha}}\left(\partial_{\alpha \dot{\alpha}}+A_{\alpha \dot{\alpha}}\right)$. Using the projection $\pi: \mathcal{P}^{6} \rightarrow \mathbb{R}^{4}$ from (3.1), we can pull back the bundle $E$ to a bundle $\mathcal{E}=\pi^{*} E$ with the pulled back connection $\mathcal{A}=\pi^{*} A$ and the covariant derivative $\tilde{\nabla}=\pi^{*} \nabla$, whose $(0,1)$-component is $^{1}$

$$
\tilde{\nabla}^{0,1}=\bar{E}^{\alpha}\left(\bar{V}_{\alpha}+\gamma \mathbf{i}_{\alpha}^{\beta} \hat{\lambda}^{\dot{\beta}} A_{\beta \dot{\beta}}\right)+\bar{E}^{3} \bar{V}_{3}
$$

\footnotetext{
${ }^{1}$ We are working on the patch $\mathcal{U}_{+}=U_{+} \times \mathbb{R}^{4} \subset \mathcal{P}^{6}$ and omit subscript and superscript "+" in formulas.
} 
Equations (2.5) of $\mathcal{J}$-holomorphic Chern-Simons theory on $\left(\mathcal{P}^{6}, \mathcal{J}\right)$ read

$$
\left[\tilde{\nabla}_{a}^{0,1}, \tilde{\nabla}_{b}^{0,1}\right]-\tilde{\nabla}_{\left[\bar{V}_{a}, \bar{V}_{b}\right]}^{0,1}=0,
$$

where $a=(\alpha, 3)=1,2,3$. Substituting (3.47) into (3.48) with

$$
\begin{aligned}
& \overline{\mathcal{A}}_{\alpha}=\gamma \dot{\mathbf{l}}_{\alpha}^{\beta} \hat{\lambda}^{\dot{\beta}} A_{\beta \dot{\beta}}, \quad \overline{\mathcal{A}}_{\alpha}=0, \\
& \mathcal{A}_{\alpha}=\gamma \lambda^{\dot{\alpha}} A_{\alpha \dot{\alpha}} \quad \text { and } \quad A_{3}=0 \text {, }
\end{aligned}
$$

we see that (3.48) are equivalent to the equations

$$
\hat{\lambda}^{\dot{\alpha}} \hat{\lambda}^{\dot{\beta}}\left[\partial_{\alpha \dot{\alpha}}+A_{\alpha \dot{\alpha}}, \partial_{\beta \dot{\beta}}+A_{\beta \dot{\beta}}\right]=\hat{\lambda}^{\dot{\alpha}} \hat{\lambda}^{\dot{\beta}} F_{\alpha \dot{\alpha}, \beta \dot{\beta}}=0,
$$

where $F=\mathrm{d} A+A \wedge A$ is the curvature of $A$. Recall that in the spinor notation $F$ has the components

$$
F_{\alpha \dot{\alpha}, \beta \dot{\beta}}=\varepsilon_{\dot{\alpha} \dot{\beta}} f_{\alpha \beta}+\varepsilon_{\alpha \beta} f_{\dot{\alpha} \dot{\beta}},
$$

where symmetric tensors

$$
f_{\alpha \beta}=\frac{1}{2} \varepsilon^{\dot{\alpha} \dot{\beta}} F_{\alpha \dot{\alpha}, \beta \dot{\beta}} \quad \text { and } \quad f_{\dot{\alpha} \dot{\beta}}=\frac{1}{2} \varepsilon^{\alpha \beta} F_{\alpha \dot{\alpha}, \beta \dot{\beta}}
$$

represent self-dual $F^{+}$and anti-self-dual $F^{-}$parts of the curvature $F=F^{+}+F^{-}$. Hence, the $\mathcal{J}$-hCS equations (3.48) on $\left(\mathcal{P}^{6}, \mathcal{J}\right)$ with $\mathcal{F}\left(V_{3}, \bar{V}_{3}\right)=0$ are equivalent to the SDYM equations on $\mathbb{R}^{4}$,

$$
F^{-}=0 \Leftrightarrow \varepsilon^{\alpha \beta} F_{\alpha \dot{\alpha}, \beta \dot{\beta}}=0,
$$

and any solution $A$ of the SDYM equations (3.53) defines a solution of the $\mathcal{J}$-hCS equations (3.48) and vice versa.

\section{TWISTOR ACTIONS FOR YANG-MILLS THEORY}

\section{A. Graded twistor space $\mathcal{P}^{6 / 2}$}

Recall that on $\mathcal{P}^{6}$ there are globally defined $(3,0)$-form $\Omega$ given by (3.42) and its complex conjugate (0,3)-form $\bar{\Omega}$. Hence, the $\mathcal{J}$-hCS action functional (1.4) is well defined on $\left(\mathcal{P}^{6}, \mathcal{J}\right)$. However, if we substitute (3.49) into (1.4) then we obtain $S=0$ since $(0,3)$-part of Chern-Simons form $\operatorname{CS}(\mathcal{A})$ on $\left(\mathcal{P}^{6}, \mathcal{J}\right)$ vanishes if $\mathcal{A}_{3}=\overline{\mathcal{A}}_{3}=0$. To obtain a nontrivial Lagrangian, one can perform a gauge transformation, which will give some nonvanishing terms ${ }^{2}$ as it was done in $[16,17]$. We will not follow this path here because this way we can at best get the actions [18-21] which have various limitations in comparison with the Chalmers-Siegel action [12].

\footnotetext{
${ }^{2}$ Chern-Simons term $\operatorname{CS}(\mathcal{A})$ is not invariant under gauge transformations.
}

The action [12] cannot be obtained without introducing additional degrees of freedom since it contains an extra propagating field $G_{\dot{\alpha} \dot{\beta}}$. One of the possibilities for introducing additional fields is to consider vector bundles $\mathcal{E}$ over $\mathcal{P}^{6}$ that are not trivial after restriction to $\mathbb{C} P^{1} \hookrightarrow \mathcal{P}^{6}$ [25]. Another possibility is to consider a graded extension of the twistor space $\left(\mathcal{P}^{6}, \mathcal{J}\right)$ similar to the cases considered by Wolf $[10,14]$ and Sämann $[9,15]$ for the complex twistor space $\mathcal{P}_{\mathbb{C}}^{3}$. We will use the second option and introduce a graded twistor space $\mathcal{P}^{6 / 2}$.

The space $\mathcal{P}^{6 \mid 2}$ is parametrized by bosonic coordinates on $\mathcal{P}^{6}$ and by two anticommuting (fermionic) coordinates $\eta_{i}$,

$$
\eta_{1} \eta_{2}+\eta_{2} \eta_{1}=0
$$

generating the Grassmann algebra

$$
\Lambda\left(\mathbb{R}^{2}\right)=\Lambda^{0}\left(\mathbb{R}^{2}\right) \oplus \Lambda^{1}\left(\mathbb{R}^{2}\right) \oplus \Lambda^{2}\left(\mathbb{R}^{2}\right)
$$

where

$\mathbf{1} \cdot \mathbb{R} \in \Lambda^{0}\left(\mathbb{R}^{2}\right), \quad \eta_{i} \in \Lambda^{1}\left(\mathbb{R}^{2}\right), \quad i=1,2 \quad$ and

$$
\eta:=\eta_{1} \eta_{2} \in \Lambda^{2}\left(\mathbb{R}^{2}\right)
$$

In the algebra (4.2) one may introduce $\mathbb{Z}_{2}$-grading,

$$
\Lambda\left(\mathbb{R}^{2}\right)=\Lambda_{0}\left(\mathbb{R}^{2}\right) \oplus \Lambda_{1}\left(\mathbb{R}^{2}\right),
$$

where

$\Lambda_{0}\left(\mathbb{R}^{2}\right)=\Lambda^{0}\left(\mathbb{R}^{2}\right) \oplus \Lambda^{2}\left(\mathbb{R}^{2}\right) \quad$ and $\quad \Lambda_{1}\left(\mathbb{R}^{2}\right)=\Lambda^{1}\left(\mathbb{R}^{2}\right)$

We set $\operatorname{gr} f=0$ if $f \in \Lambda_{0}\left(\mathbb{R}^{2}\right)$ and $\operatorname{gr} f=1$ if $f \in \Lambda_{1}\left(\mathbb{R}^{2}\right)$, $\operatorname{gr} f$ is the Grassmann parity of $f$.

On the space $\mathcal{P}^{6}$ we consider the space $\mathrm{Gr}_{\mathcal{P}^{6}}$ of locally defined functions (a sheaf) with values in the Grassmann algebra $\Lambda\left(\mathbb{R}^{2}\right)$. A manifold $\mathcal{P}^{6}$ with the sheaf $\mathrm{Gr}_{\mathcal{P}^{6}}$ is a graded manifold $\mathcal{P}^{6 / 2}=\left(\mathcal{P}^{6}, \operatorname{Gr}_{\mathcal{P}^{6}}\right)[26,27]$ that can be viewed as the trivial bundle $\mathcal{P}^{6} \times \Lambda_{1}\left(\mathbb{R}^{2}\right) \rightarrow \mathcal{P}^{6}$. Tangent spaces of $\mathcal{P}^{6 / 2}$ are defined by the even vector fields (3.33), (3.34) together with the odd vector fields

$$
\partial^{i}:=\frac{\partial}{\partial \eta_{i}} \quad \text { such that } \frac{\partial}{\partial \eta_{1}} \frac{\partial}{\partial \eta_{2}}+\frac{\partial}{\partial \eta_{2}} \frac{\partial}{\partial \eta_{1}}=0
$$

commuting with the even vector fields on $\mathcal{P}^{6}$. Respectively, the space of differential forms on $\mathcal{P}^{6 / 2}$ has the local basis $\left\{E^{a}, \bar{E}^{a}, \mathrm{~d} \eta_{i}\right\}$ with commutation relations 


$$
\begin{aligned}
\mathrm{d} \eta_{1} \wedge \mathrm{d} \eta_{2} & =\mathrm{d} \eta_{2} \wedge \mathrm{d} \eta_{1}, \\
E^{a} \wedge \mathrm{d} \eta_{i} & =\mathrm{d} \eta_{i} \wedge E^{a} \quad \text { and } \quad \bar{E}^{a} \wedge \mathrm{d} \eta_{i}=\mathrm{d} \eta_{i} \wedge \bar{E}^{a}
\end{aligned}
$$

where $\left\{E^{a}, \bar{E}^{a}\right\}$ are given in (3.35).

Recall that on $\left(\mathcal{P}^{6}, \mathcal{J}\right)$ there are globally defined forms $\Omega$ and $\bar{\Omega}$. Hence, on $\mathcal{P}^{6 / 2}$ we can introduce a closed (3|2)form

$$
\operatorname{Im} \Omega \wedge \mathrm{d} \eta_{1} \wedge \mathrm{d} \eta_{2}
$$

and the volume form

$$
\frac{\mathrm{i}}{8} \Omega \wedge \bar{\Omega} \wedge \mathrm{d} \eta=-\frac{\mathrm{i}}{2} \mathrm{~d}^{4} x \wedge \frac{\mathrm{d} \lambda \wedge \mathrm{d} \bar{\lambda}}{(1+\lambda \bar{\lambda})^{2}} \wedge \mathrm{d} \eta,
$$

where $\mathrm{d} \eta=\mathrm{d} \eta_{1} \wedge \mathrm{d} \eta_{2}$.

\section{B. Chern-Simons type theory on $\mathcal{P}^{6 \mid 2}$}

Let $\mathcal{E}$ be a trivial rank $k$ complex vector bundle over $\mathcal{P}^{6 / 2}$ and $\mathcal{A}$ a connection one-form on $\mathcal{E}$. We choose the connection $\mathcal{A}$ depending on all coordinates on $\mathcal{P}^{6 / 2}$ and having no components along the Grassmann directions. The curvature $\mathcal{F}$ of such $\mathcal{A}$ is

$$
\mathcal{F}=\mathcal{F}^{\mathrm{B}}+\mathcal{F}^{\mathrm{F}}=\mathrm{d}^{\mathrm{B}} \mathcal{A}+\mathcal{A} \wedge \mathcal{A}+\mathrm{d}^{\mathrm{F}} \mathcal{A},
$$

where $\mathrm{d}^{\mathrm{B}}$ is the bosonic part (3.36) of the exterior derivative $\mathrm{d}=\mathrm{d}^{\mathrm{B}}+\mathrm{d}^{\mathrm{F}}$ and

$$
\mathrm{d}^{\mathrm{F}}=\mathrm{d} \eta_{i} \partial^{i} \quad \text { for } \quad \partial^{i}=\frac{\partial}{\partial \eta_{i}}
$$

is the fermionic part of $\mathrm{d}$.

Consider the action functional

$$
S=\int_{\mathcal{P}^{6 \mid 2}} \operatorname{Im} \Omega \wedge \mathrm{d} \eta \wedge \mathrm{CS}(\mathcal{A}),
$$

where

$$
\mathrm{CS}(\mathcal{A})=\operatorname{tr}\left(\mathcal{A} \wedge \mathrm{d}^{\mathrm{B}} \mathcal{A}+\frac{2}{3} \mathcal{A} \wedge \mathcal{A} \wedge \mathcal{A}\right)
$$

is the Chern-Simons 3-form. Field equations following from (4.12) read

$$
\operatorname{Im} \Omega \wedge \mathcal{F}^{\mathrm{B}}=0
$$

where $\mathcal{F}^{\mathrm{B}}$ is defined in (4.10). From (4.14) it follows that

$$
\operatorname{Re} \Omega \wedge \mathcal{F}^{\mathrm{B}}=0
$$

since $\Omega$ is a (3,0)-form with respect to $\mathcal{J}$,

$$
\mathcal{J} \Omega=\mathrm{i} \Omega \Leftrightarrow \mathcal{J} \operatorname{Im} \Omega=\operatorname{Re} \Omega .
$$

Combining (4.14) and (4.15), we obtain

$$
\Omega \wedge \mathcal{F}_{\mathrm{B}}^{0,2}=0 \Leftrightarrow \mathcal{F}_{\mathrm{B}}^{0,2}=0 .
$$

Note that from (3.45) and (4.17) it follows that [28,29]

$$
\mathcal{F}^{\mathrm{B}}\left(V_{1}, \bar{V}_{1}\right)+\mathcal{F}^{\mathrm{B}}\left(V_{2}, \bar{V}_{2}\right)=0 .
$$

The action functional (4.12) and solution to the equations (4.14)-(4.17) were considered in [7,28,29].

\section{Field equations on $\mathcal{P}^{6}$}

Having given necessary ingredients, we may now consider $\mathcal{J}$-hCS field equations (4.17). These equations on the patch $\hat{\mathcal{U}}_{+}=\mathcal{U}_{+} \times \Lambda_{1}\left(\mathbb{R}^{2}\right)$ of $\mathcal{P}^{6 / 2}$ read

$$
\begin{aligned}
& \bar{V}_{\alpha} \overline{\mathcal{A}}_{\beta}-\bar{V}_{\beta} \overline{\mathcal{A}}_{\alpha}+\left[\overline{\mathcal{A}}_{\alpha}, \overline{\mathcal{A}}_{\beta}\right]=0, \\
& \left.\bar{V}_{3} \overline{\mathcal{A}}_{\alpha}-\bar{V}_{\alpha} \overline{\mathcal{A}}_{3}+\left[\overline{\mathcal{A}}_{3}, \overline{\mathcal{A}}_{\alpha}\right]-\left[\bar{V}_{3}, \bar{V}_{\alpha}\right]\right\lrcorner \mathcal{A}=0,
\end{aligned}
$$

where "״" denotes the interior product of vector fields with differential forms. Here we used components of $\mathcal{A}$ in the expansion

$$
\mathcal{A}=\mathcal{A}_{a} E^{a}+\overline{\mathcal{A}}_{a} \bar{E}^{a}=\mathcal{A}_{\alpha} E^{\alpha}+\mathcal{A}_{3} E^{3}+\overline{\mathcal{A}}_{\alpha} \bar{E}^{\alpha}+\overline{\mathcal{A}}_{3} \bar{E}^{3} .
$$

As usual in the twistor approach, we work in a gauge in which $\overline{\mathcal{A}}_{3} \neq 0$ but the bosonic part of $\overline{\mathcal{A}}_{3}$ is zero. Note that in general the gauge potential $\mathcal{A}$ in (4.18) and (4.19) can be expanded in the odd coordinates $\eta_{i}$ as

$$
\mathcal{A}=A+\eta_{i} \psi^{i}+\eta_{1} \eta_{2} G \text {. }
$$

For simplicity and more clarity we first consider the truncated case $\psi^{i}=0$ and discuss the case $\psi^{i} \neq 0$ afterwards.

\section{Remark}

The connection (4.20) on the vector bundle $\mathcal{E}$ over $\mathcal{P}^{6 \mid 2} \cong \mathcal{P}^{6} \times \Lambda_{1}\left(\mathbb{R}^{2}\right)$ takes values in the Lie algebra $\mathfrak{g}$ of a semisimple Lie group $G$. Note that maps from the space $\Lambda_{1}\left(\mathbb{R}^{2}\right)$ in (4.5) to the group $G$ form a supergroup super- $T G$ [30], where $T G=G \ltimes \mathfrak{g}$ is the semidirect product of $G$ and $\mathfrak{g}$. That is why the field $\mathcal{A}$ in (4.20) can be considered as a connection on a super- $T G$ bundle $\mathcal{E}^{\prime}$ over the bosonic twistor space $\mathcal{P}^{6}$. This kind of correspondence was found by Witten when studying Chern-Simons theories on 3manifolds [30].

From (3.33)-(3.35) one concludes that components $\overline{\mathcal{A}}_{\alpha}$ and $\overline{\mathcal{A}}_{3}$ take values in the bundles $\mathcal{O}(-1)$ and $\mathcal{O}(2)$ over $\mathbb{C} P^{1}$ and $\mathcal{A}_{\alpha}$ and $\mathcal{A}_{3}$ take values in the complex conjugate bundles. This fixes the dependence of $\mathcal{A}$ on $\lambda$ and $\bar{\lambda}$ up to a 
gauge transformations (cf. [9-11,14,15]). Namely, we obtain

$$
\begin{aligned}
& \mathcal{A}_{\alpha}=\gamma\left\{\lambda^{\dot{\alpha}} A_{\alpha \dot{\alpha}}+\eta\left(\lambda^{\dot{\alpha}} G_{\alpha \dot{\alpha}}+\gamma \lambda^{\dot{\alpha}} \hat{\lambda}^{\dot{\beta}} \lambda^{\dot{\gamma}} G_{\alpha \dot{\alpha} \dot{\beta} \dot{\gamma}}\right)\right\} \\
& =\gamma\left\{\lambda^{\dot{\alpha}}\left(A_{\alpha \dot{\alpha}}+\eta B_{\alpha \dot{\alpha}}\right)+\eta \gamma \lambda^{\left(\dot{\alpha} \hat{\lambda}^{\dot{\beta}} \lambda^{\dot{\gamma})}\right.} G_{\alpha(\dot{\alpha} \dot{\beta} \dot{\gamma})}\right\}, \\
& \overline{\mathcal{A}}_{\alpha}=\gamma \dot{\mathbf{j}}_{\alpha}^{\beta}\left\{\hat{\lambda}^{\dot{\beta}} A_{\beta \dot{\beta}}+\eta\left(\hat{\lambda}^{\dot{\beta}} G_{\beta \dot{\beta}}+\gamma \hat{\lambda}^{\dot{\beta}} \lambda^{\dot{\gamma}} \hat{\lambda}^{\dot{\sigma}} G_{\beta \dot{\beta} \dot{\gamma} \dot{\sigma}}\right)\right\} \\
& \left.=\gamma \mathbf{i}_{\alpha}^{\dot{\beta}}\left\{\hat{\lambda}^{\dot{\beta}}\left(A_{\beta \dot{\beta}}+\eta B_{\beta \dot{\beta}}\right)+\eta \gamma \hat{\lambda}^{(\dot{\beta}} \lambda^{\dot{\gamma}} \hat{\lambda}^{\dot{\sigma})} G_{\beta(\dot{\beta} \dot{\gamma} \dot{\sigma})}\right)\right\}, \\
& \mathcal{A}_{3}=\eta \hat{\lambda}^{\dot{\beta}} \hat{\lambda}^{\dot{\gamma}} G_{\dot{\beta} \dot{\gamma}} \quad \text { and } \quad \overline{\mathcal{A}}_{3}=-\eta \lambda^{\dot{\beta}} \lambda^{\dot{\gamma}} G_{\dot{\beta} \dot{\gamma}} \text {, }
\end{aligned}
$$

where

$$
B_{\alpha \dot{\alpha}}:=G_{\alpha \dot{\alpha}}-\frac{1}{3} \varepsilon^{\dot{\beta} \dot{\gamma}}\left(G_{\alpha \dot{\alpha} \dot{\beta} \dot{\gamma}}-G_{\alpha \dot{\beta} \dot{\gamma} \dot{\alpha}}\right)
$$

and the coefficient fields $A_{\alpha \dot{\alpha}}, G_{\alpha \dot{\alpha}}, \ldots$ do only depend on $x^{\alpha \dot{\alpha}} \in \mathbb{R}^{4}$. Here $\lambda^{\dot{\alpha}}, \hat{\lambda}^{\dot{\alpha}}$ are given in (3.29) and (3.32), $\eta=\eta_{1} \eta_{2}$, and parentheses denote normalized symmetrization with respect to the enclosed indices.

Substituting (4.21) into (4.18), we obtain the equations

$$
G_{\alpha(\dot{\alpha} \dot{\beta} \dot{\gamma})}=\nabla_{\alpha(\dot{\alpha}} G_{\dot{\beta} \dot{\gamma})},
$$

showing that $G_{\alpha(\dot{\alpha} \dot{\beta} \dot{\gamma})}$ are composite fields describing no independent degrees of freedom. Other nontrivial equations following from (4.18) after substituting (4.21) read

$$
\begin{gathered}
\varepsilon^{\alpha \beta}\left[\partial_{\alpha \dot{\alpha}}+A_{\alpha \dot{\alpha}}, \partial_{\beta \dot{\beta}}+A_{\beta \dot{\beta}}\right]=\varepsilon^{\alpha \beta} F_{\alpha \dot{\alpha}, \beta \dot{\beta}}=0, \\
\varepsilon^{\alpha \beta} \nabla_{\alpha \dot{\alpha}} B_{\beta \dot{\beta}}=0, \\
\varepsilon^{\dot{\alpha} \dot{\beta}} \nabla_{\alpha \dot{\alpha}} G_{\dot{\beta} \dot{\gamma}}=0 .
\end{gathered}
$$

We see that (4.24) coincide with the SDYM equations on $\mathbb{R}^{4}$ and (4.25) are the linearized SDYM equations for

$$
\delta A_{\beta \dot{\beta}}=B_{\beta \dot{\beta}} .
$$

Hence, $B_{\alpha \dot{\alpha}}$ is a tangent vector at $A_{\alpha \dot{\alpha}}$ to the solution space of the SDYM equations. It is a secondary field (a symmetry) depending on $A_{\alpha \dot{\alpha}}$ and for simplicity we neglect it by choosing $B_{\alpha \dot{\alpha}}=0$. The rest equations (4.24) and (4.26) are the Chalmers-Siegel equations describing the self-dual gauge potential $A_{\alpha \dot{\alpha}}$ and the anti-self-dual field $G_{\alpha \dot{\alpha}, \beta \dot{\beta}}=$ $\varepsilon_{\alpha \beta} G_{\dot{\alpha} \dot{\beta}}$ propagating in the self-dual background.

The action functional associated with (4.24) and (4.26) is given by

$$
S_{\mathrm{sd}}=2 \int_{\mathbb{R}^{4}} \mathrm{~d}^{4} x \operatorname{tr}\left(G^{\dot{\alpha} \dot{\beta}} f_{\dot{\alpha} \dot{\beta}}\right)
$$

with $f_{\dot{\alpha} \dot{\beta}}$ given by (3.52). This action can be obtained from (4.12) after splitting,

$$
\mathcal{A}=X+\eta Y
$$

into ordinary bosonic and even nilpotent parts, using the formula $^{3}$

$\mathrm{CS}(X+\eta Y)=\mathrm{CS}(X)+2 \eta \operatorname{tr}(Y \wedge \mathcal{F}(X))-\eta \mathrm{d}^{\mathrm{B}}(\operatorname{tr}(X \wedge Y))$

and integrating over the nilpotent coordinate $\eta$ and over $\mathbb{C} P^{1} \hookrightarrow \mathcal{P}^{6 / 2}$.

\section{E. Extra terms}

As we mentioned earlier, the general expansion (4.20) of connection $\mathcal{A}$ in odd coordinates $\eta_{i}$ contains fermionic fields $\psi^{i}\left(x^{\alpha \dot{\alpha}}\right)$ which we consider now. Expansion (4.20) can be written in components as

$\mathcal{A}_{\alpha}=\gamma \lambda^{\dot{\alpha}} A_{\alpha \dot{\alpha}}\left(\eta_{1}, \eta_{2}\right) \quad$ and $\quad \mathcal{A}_{3}=\hat{\lambda}^{\dot{\alpha}} \hat{\lambda}^{\dot{\beta}} G_{\dot{\alpha} \dot{\beta}}\left(\eta_{1}, \eta_{2}\right)$,

where

$$
\begin{array}{r}
A_{\alpha \dot{\alpha}}\left(\eta_{1}, \eta_{2}\right)= \\
A_{\alpha \dot{\alpha}}+\eta_{i}\left(\psi_{\alpha \dot{\alpha}}^{i}+\gamma \hat{\lambda}^{\dot{\beta}} \lambda^{\dot{\lambda}} \psi_{\alpha(\dot{\alpha} \dot{\beta} \dot{\gamma})}^{i}\right) \\
+\eta_{1} \eta_{2}\left(B_{\alpha \dot{\alpha}}+\gamma \hat{\lambda}^{\dot{\beta}} \lambda^{\dot{\gamma}} G_{\alpha(\dot{\alpha} \dot{\beta} \dot{\gamma})}\right), \\
G_{\dot{\alpha} \dot{\beta}}\left(\eta_{1}, \eta_{2}\right)=\eta_{i} \psi_{\dot{\alpha} \dot{\beta}}^{i}+\eta_{1} \eta_{2} G_{\dot{\alpha} \dot{\beta}} .
\end{array}
$$

For $\overline{\mathcal{A}}_{\alpha}$ and $\overline{\mathcal{A}}_{3}$ we have

$\overline{\mathcal{A}}_{\alpha}=\gamma \dot{\mathbf{j}} \dot{\alpha}_{\alpha}^{\beta} \hat{\lambda}^{\dot{\beta}} A_{\beta \dot{\beta}}\left(\eta_{1}, \eta_{2}\right) \quad$ and $\quad \overline{\mathcal{A}}_{3}=-\lambda^{\dot{\alpha}} \lambda^{\dot{\beta}} G_{\dot{\alpha} \dot{\beta}}\left(\eta_{1}, \eta_{2}\right)$.

Substituting (4.31)-(4.34) into the Eqs. (4.18), we obtain the Eqs. (4.23)-(4.27) and additional equations

$$
\begin{gathered}
\varepsilon^{\alpha \beta} \nabla_{\alpha \dot{\alpha}} \psi_{\beta \dot{\beta}}^{1}=0 \quad \text { and } \quad \psi_{\beta \dot{\beta}}^{2}=0, \\
\varepsilon^{\dot{\alpha} \dot{\beta}} \nabla_{\alpha \dot{\alpha}} \psi_{\dot{\beta} \dot{\gamma}}^{1}=0 \quad \text { and } \quad \psi_{\alpha(\dot{\alpha} \dot{\beta} \dot{\gamma})}^{1}=\nabla_{\alpha(\dot{\alpha}} \psi_{\dot{\beta} \dot{\gamma})}^{1}, \\
\psi_{\dot{\beta} \dot{\gamma}}^{2}=0 \quad \text { and } \quad \psi_{\alpha(\dot{\alpha} \dot{\beta} \dot{\gamma})}^{2}=\nabla_{\alpha(\dot{\alpha}} \psi_{\dot{\beta} \dot{\gamma})}^{2}=0 .
\end{gathered}
$$

From (4.25) and (4.35) we see that $B_{\alpha \dot{\alpha}}$ and $\psi_{\alpha \dot{\alpha}}^{1}$ are even and odd solutions of the linearized SDYM equations and $\psi_{\dot{\beta} \dot{\gamma}}^{1}$ in (4.36) is an odd solution to the linearized form of equation (4.26) for $\delta G_{\dot{\beta} \dot{\gamma}}$. Thus, the general form (4.20) of $\mathcal{A}$ reduces the $\mathcal{J}$-hCS equations (4.18) to the Chalmers-

\footnotetext{
${ }^{3}$ Recall that in all formulas here $\mathrm{d}^{\mathrm{B}}$ is the ordinary bosonic exterior derivative.
} 
Siegel equations (4.24) and (4.26) together with their linearized form, solutions of which describe even and odd tangent vectors to the solution space.

\section{F. Full Yang-Mills}

So far, we have shown that the Chalmers-Siegel action (4.28) for SDYM theory can be obtained from the ChernSimons type action (4.12) on the graded twistor space $\mathcal{P}^{6 / 2}$. It is known that the action (4.28) is a limit of the full YangMills action for small coupling constant $g_{\mathrm{YM}}$. Namely, let us modify the action (4.28) by adding the term

$$
S_{\varepsilon}=-\varepsilon^{2} \int_{\mathbb{R}^{4}} \mathrm{~d}^{4} x \operatorname{tr}\left(G^{\dot{\alpha} \dot{\beta}} G_{\dot{\alpha} \dot{\beta}}\right),
$$

so that

$S_{\text {tot }}=S_{\mathrm{sd}}+S_{\varepsilon}=2 \int_{\mathbb{R}^{4}} \mathrm{~d}^{4} x \operatorname{tr}\left(G^{\dot{\alpha} \dot{\beta}} f_{\dot{\alpha} \dot{\beta}}-\frac{1}{2} \varepsilon^{2} G^{\dot{\alpha} \dot{\beta}} G_{\dot{\alpha} \dot{\beta}}\right)$.

Here $\varepsilon$ is some small parameter. Variation of $S_{\text {tot }}$ with respect to $G_{\dot{\alpha} \dot{\beta}}$ gives

$$
G_{\dot{\alpha} \dot{\beta}}=\frac{1}{\varepsilon^{2}} f_{\dot{\alpha} \dot{\beta}}
$$

Substituting (4.40) back into (4.39), we obtain

$$
\begin{aligned}
S_{\mathrm{tot}} & =\frac{1}{\varepsilon^{2}} \int_{\mathbb{R}^{4}} \mathrm{~d}^{4} x \operatorname{tr}\left(f_{\dot{\alpha} \dot{\beta}} f^{\dot{\alpha} \dot{\beta}}\right)=\frac{1}{2 \varepsilon^{2}} \int_{\mathbb{R}^{4}} \operatorname{tr}\left(F^{-} \wedge F^{-}\right) \\
& =-\frac{1}{4 \varepsilon^{2}} \int_{\mathbb{R}^{4}} \operatorname{tr}(F \wedge * F)+\frac{1}{4 \varepsilon^{2}} \int_{\mathbb{R}^{4}} \operatorname{tr}(F \wedge F) .
\end{aligned}
$$

Hence, the action (4.41) is equivalent to the standard YangMills action

$$
S_{\mathrm{YM}}=-\frac{1}{4 g_{\mathrm{YM}}^{2}} \int_{\mathbb{R}^{4}} \operatorname{tr}(F \wedge * F),
$$

with the coupling constant $g_{\mathrm{YM}}=\varepsilon$, plus the topological term. Therefore, for obtaining the Yang-Mills action (4.42) we should derive the term (4.38) from the twistor space.

\section{G. Twistor action for full Yang-Mills}

Recall that $\eta=\eta_{1} \eta_{2}$, where $\eta_{1}$ and $\eta_{2}$ are real Grassmann variables. Consider a connection $\mathcal{A}$ depending on $\eta$ as written in (4.21). ${ }^{4}$ It does not have components along the

\footnotetext{
${ }^{4}$ We do not consider the more general dependence (4.31)(4.34) since we only want to show that one can obtain the action (4.42) from the twistor space. Consideration of (4.31)-(4.34) will give the Yang-Mills theory with its infinitesimal symmetries as we saw in the case of the SDYM equations.
}

Grassmann directions but the mixed components of the curvature,

$$
\begin{aligned}
\mathcal{F}^{\mathrm{F}} & =\mathrm{d}^{\mathrm{F}} \mathcal{A}=\left(\partial^{i} \mathcal{A}_{a}\right) \mathrm{d} \eta_{i} \wedge E^{a}+\left(\partial^{i} \overline{\mathcal{A}}_{a}\right) \mathrm{d} \eta_{i} \wedge \bar{E}^{a} \\
& =\mathcal{F}_{a}^{i} \mathrm{~d} \eta_{i} \wedge E^{a}+\overline{\mathcal{F}}_{a}^{i} \mathrm{~d} \eta_{i} \wedge \bar{E}^{a},
\end{aligned}
$$

do not vanish. In particular, for restriction of $\mathcal{F}^{\mathrm{F}}$ to $\mathbb{C} P^{1 / 2} \hookrightarrow \mathcal{P}^{6 / 2}$ we have

$$
\mathcal{F}_{\mid C P^{1 / 2}}^{\mathrm{F}}=\mathcal{F}_{\lambda}^{i} \mathrm{~d} \eta_{i} \wedge \mathrm{d} \lambda+\mathcal{F}_{\bar{\lambda}}^{i} \mathrm{~d} \eta_{i} \wedge \mathrm{d} \bar{\lambda}
$$

where

$\mathcal{F}_{\lambda}^{i}=-\varepsilon^{i j} \eta_{j} \gamma^{2} \hat{\lambda}^{\dot{\alpha}} \hat{\lambda}^{\dot{\beta}} G_{\dot{\alpha} \dot{\beta}} \quad$ and $\quad \mathcal{F}_{\bar{\lambda}}^{i}=\varepsilon^{i j} \eta_{j} \gamma^{2} \lambda^{\dot{\alpha}} \lambda^{\dot{\beta}} G_{\dot{\alpha} \dot{\beta}}$

Using (4.45), we can introduce the gauge invariant functional

$$
\frac{\mathrm{i} \varepsilon^{2}}{8} \int_{\mathcal{P}^{6 \mid 2}} \Omega \wedge \bar{\Omega} \wedge \mathrm{d} \eta_{1} \wedge \mathrm{d} \eta_{2} \varepsilon_{i j} g^{\lambda \bar{\lambda}} \operatorname{tr}\left(\mathcal{F}_{\lambda}^{i} \mathcal{F}_{\bar{\lambda}}^{j}\right)
$$

where

$$
\mathrm{d} s_{\mathbb{C} P^{1}}^{2}=g_{\lambda \bar{\lambda}} E^{\lambda} \otimes E^{\bar{\lambda}} \Leftrightarrow g_{\lambda \bar{\lambda}}=\gamma^{2} \text { and } g^{\lambda \bar{\lambda}}=\gamma^{-2} .
$$

Integrating $\operatorname{tr}\left(\mathcal{F}_{\lambda}^{i} \mathcal{F}_{\bar{\lambda}}^{j}\right)$ in (4.46) over fermionic coordinates and over $\mathbb{C} P^{1} \hookrightarrow \mathcal{P}^{6 \mid 2}$, we obtain the functional $S_{\varepsilon}$ given by (4.38). Hence, adding the local term given by (4.46) to the Chern-Simons type Lagrangian in (4.12), we obtain the full Yang-Mills action (4.42).

\section{CONCLUSIONS}

In this paper we considered graded twistor space $\mathcal{P}^{6 / 2}$ with a nonintegrable almost complex structure $\mathcal{J}$ and $\mathcal{J}$-holomorphic Chern-Simons theory on $\mathcal{P}^{6 \mid 2}$. It was shown that under some assumptions this theory is equivalent to self-dual Yang-Mills theory on $\mathbb{R}^{4}$. In our discussion we tried to be close to the consideration of the papers [14,15], where $\mathcal{N}<4$ SDYM theories were derived from holomorphic Chern-Simons theory on complex supertwistor spaces. We have also shown that the full Yang-Mills action in $\mathbb{R}^{4}$ can be obtained from a twistor action on $\mathcal{P}^{6 / 2}$ with a locally defined Lagrangian. We did not pursue the goal of studying all these tasks in full generality. We wanted to show the principal possibility of obtaining actions for Yang-Mills and its selfdual subsector from a twistor action. Examining all aspects of the model requires additional efforts.

\section{ACKNOWLEDGMENTS}

This work was supported by the Deutsche Forschu ngsgemeinschaft Grant No. LE 838/19. 
[1] M. F. Atiyah, N. J. Hitchin, and I. M. Singer, Self-duality in four-dimensional Riemannian geometry, Proc. R. Soc. A 362, 425 (1978).

[2] J. Eells and S. Salamon, Constructions twistorielles des applications harmoniques, C. R. Acad. Sci. Paris 296, 685 (1983).

[3] R. Penrose and W. Rindler, Spinors and space-time (Cambridge University Press, Cambridge, England, 1984\&1985), Vols. 1\&2.

[4] R. S. Ward and R. O. Wells, Twistor Geometry and Field Theory (Cambridge University Press, Cambridge, England, 1990).

[5] L. J. Mason and N. M. J. Woodhouse, Integrability, SelfDuality, and Twistor Theory (Clarendon Press, Oxford, 1996).

[6] F. Xu, Geometry of SU(3) manifolds, Ph.D. thesis, Duke University, 2008.

[7] A. D. Popov, Hermitian-Yang-Mills equations and pseudoholomorphic bundles on nearly Kähler and nearly CalabiYau twistor 6-manifolds, Nucl. Phys. B828, 594 (2010).

[8] E. Witten, Perturbative gauge theory as a string theory in twistor space, Commun. Math. Phys. 252, 189 (2004).

[9] C. Sämann, Aspects of twistor geometry and supersymmetric field theories within superstring theory, arXiv:hep-th/ 0603098.

[10] M. Wolf, On supertwistor geometry and integrability in super gauge theory, arXiv:hep-th/0611013.

[11] M. Wolf, A first course on twistors, integrability and gluon scattering amplitudes, J. Phys. A 43, 393001 (2010).

[12] G. Chalmers and W. Siegel, The self-dual sector of QCD amplitudes, Phys. Rev. D 54, 7628 (1996).

[13] O. Lechtenfeld and A. D. Popov, Supertwistors and cubic string field theory for open $\mathcal{N}=2$ strings, Phys. Lett. B 598, 113 (2004).

[14] M. Wolf, Topological B-model on weighted projective spaces and self-dual models in four dimensions, J. High Energy Phys. 09 (2004) 007.

[15] C. Sämann, The topological B-model on fattened complex manifolds and subsectors of $\mathcal{N}=4$ self-dual Yang-Mills theory, J. High Energy Phys. 01 (2005) 042.
[16] K. Costello, Topological strings, twistors, and Skyrmions, Available at http://web.math.ucsb.edu/WHCGP/.

[17] R. Bittleston and D. Skinner, Twistors, the ASD YangMills equations, and $4 d$ Chern-Simons theory, arXiv:2011 .04638 .

[18] S. K. Donaldson, Anti-self-dual Yang-Mills connections over complex algebraic surfaces and stable vector bundles, Proc. London Math. Soc. s3-50, 1 (1985).

[19] V. P. Nair and J. Schiff, A Kähler-Chern-Simons theory and quantization of instanton moduli spaces, Phys. Lett. B 246, 423 (1990).

[20] A. N. Leznov and M. A. Mukhtarov, Deformation of algebras and solution of self-duality equation, J. Math. Phys. (N.Y.) 28, 2574 (1987).

[21] A. Parkes, A cubic action for self-dual Yang-Mills, Phys. Lett. B 286, 265 (1992).

[22] N. M. J. Woodhouse, Real methods in twistor theory, Classical Quantum Gravity 2, 257 (1985).

[23] R. L. Bryant, On the geometry of almost complex 6manifolds, Asian J. Math. 10, 561 (2006).

[24] S. Kobayashi and K. Nomizu, Foundations of Differential Geometry (Interscience Publishers, New York, 1969), Vol. 2.

[25] J. Leiterer, The Penrose transform for bundles non-trivial on the general line, Mathematische Nachrichten 112, 35 (1983).

[26] B. Kostant, Graded manifolds, graded Lie theory and prequantization, Lect. Notes Math. 570, 177 (1977).

[27] F. A. Berezin, Introduction to Superanalysis (Reidel, Dordrecht, 1987).

[28] D. Harland, T. A. Ivanova, O. Lechtenfeld, and A. D. Popov, Yang-Mills flows on nearly Kähler manifolds and $G_{2}$-instantons, Commun. Math. Phys. 300, 185 (2010).

[29] S. Bunk, O. Lechtenfeld, A. D. Popov, and M. Sperling, Instantons on conical half-flat 6-manifolds, J. High Energy Phys. 01 (2015) 030.

[30] E. Witten, Topology changing amplitudes in $(2+1)$-dimensional gravity, Nucl. Phys. B323, 113 (1989). 